\title{
Optimization Design of Cucumber Harvesting Manipulator by TRIZ
}

\author{
Guohua Gao a, Shuai Mab \\ School of Mechanical Engineering and Applied Electronics Technology, Beijing University of \\ Technology, Beijing 100124, China; \\ aggh6768@126.com, b1164635991@qq.com
}

Keywords: Cucumber harvesting manipulator, functional model, TRIZ, kinematic simulation.

\begin{abstract}
In this paper, the design of cucumber harvesting manipulator is completed by using the mechanism instead of the joint type design on the basis of horizontal cucumber planting mode. For the harvesting manipulator, the kinematics simulation of the mechanism is analyzed carried out by Adams. Based on the simulation results, stability problem in the mechanism harvesting manipulator was found. In order to improve of harvesting manipulator, this paper would use TRIZ to analysis solve the problem in existing design, and proposed the innovation solution to optimize it.
\end{abstract}

\section{Introduction}

Harvesting robot design is always a hot research field. Many researchers both at home and abroad have studied Harvesting robot deeply. Currently, the researchers mainly focus their study on the trajectory planning, motion solution, motion control, and flexible grasp of the end effector. However, the average success rate, damage rate and picking period of current harvesting robot were respectively $66 \%, 5 \%, 33 \mathrm{~S}$ [1]. It is difficult to put harvesting robots into application. In the other hand, the joint harvesting manipulator also raises the difficulty of mechanism design and the cost of manufacturing. So it is very necessary to develop a kind of cucumber harvesting manipulator to solve the existing problems and defects.

In order to improve the working performance of harvesting robots and reduce the cost, our group designed a harvesting for cucumber by using mechanism combination instead of the joint. And according to the manipulator design, we plan to make the kinematics simulation of mechanism by Adams so as to get the problem. As a special problem solving method which is widely applied in industrial field as well as other fields, this paper would use TRIZ to solve the found problem from simulation in existing system, and proposed the innovation solution to optimize it.

\section{Design and kinematic simulation of cucumber harvesting manipulator}

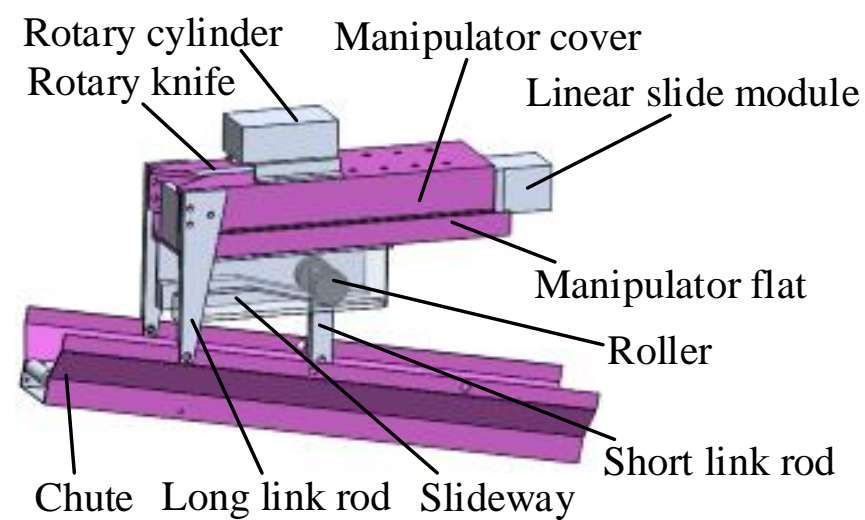

Fig. 1 The cucumber harvesting manipulator

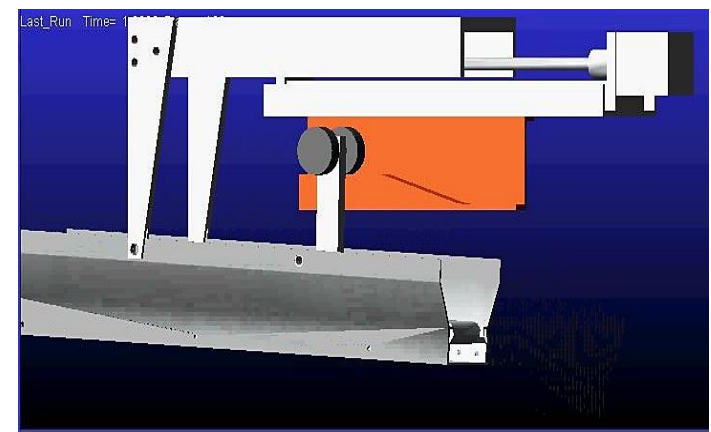

Fig. 2 Simulation model of cucumber picking manipulator by Adams

Based on the horizontal cucumber planting mode, our group finished the design of cucumber harvesting manipulator, as shown in Fig. 1. With the driving of linear slide module, manipulator cover stretches to reach the target cucumber. At the same time, chute will stretch also and be in the horizontal 
position by the limitation of slideway. The target cucumber will tilt owing to the push of chute. Next, the rotary knife will rotate and finish the cutting for stem of cucumber. With the help of gravity, the picked cucumber falls into the chute. Finally, manipulator cover makes the chute withdraw. Under the limitation and gravity as well, the slideway will tilt and the cucumber will slid back for collecting.

In order to prove the feasibility of the cucumber harvesting manipulator, this paper carried out the mechanism kinematic simulation, as shown in Fig. 2. In the simulation result, the cucumber harvesting manipulator can work as expected performance. However, we found also that the chute has a larger swing during the cover stretches of manipulator to reach the target cucumber. This swing will reduce stability of harvesting manipulator and the success rate of harvesting. As shown in Fig. 3, the peak value of swing angle of chute is $0.8358^{\circ}$, and the amplitude of the end of chute is $10 \mathrm{~mm}$. So it is essential to solve the problem to improve the stability.

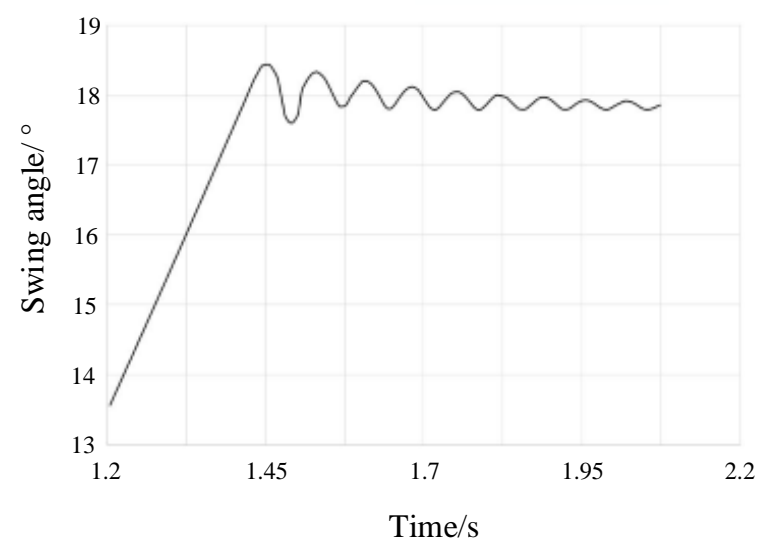

Fig. 3 The swing angle of chute in cucumber harvesting manipulator

\section{Problem Solving by TRIZ theory}

\subsection{Overview of TRIZ Theory.}

TRIZ, an acronym for the Theory of Inventive Problem Solving, began in 1946 when Altshuller, a mechanical engineer, began to study 2.5 million high level patents in the Russian Navy [2]. The core of the inventive problem in TRIZ theory is to solve the contradiction. In order to guide the solution of contradiction, Altshuller went on to classify the technical contradiction into 39 parameters [3]. Moreover, the 40 invention principles and separation principle was purposed to solve the technical contradiction problem, and establish corresponding relationship between the 39 engineering parameters and the 40 common inventive principles, which is called the Technical Contradiction Matrix [4]. The solver can look for the inventive principles to solve the problem in the Technical Contradiction Matrix on the basis of the two contradiction parameters in the system, and solve the problem with the elect inventive principles.

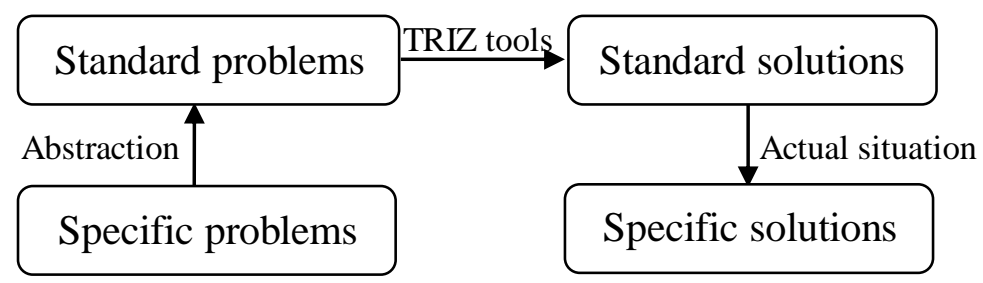

Fig. 4 The flowchart of problem solving by TRIZ

As shown in Fig. 4, the using process of TRIZ theory for problem solving can be divided into three steps: Firstly, the specific problem are bstracted into the standard problem in TRIZ. Then, the standard problem are transformed into the standard solution by TRIZ tools. Finally, the spcific solution of the problem is obtained the standard solution by considering the actual situation [5]. 


\subsection{Functional Model Analysis of Problem.}

In TRIZ theory, the functional model can help engineers understand the problem system deeply and find out the key piont of the problem. So the functional model analysis is used usually before starting to solve the problem.

For the stability problem of chute swing, we set the functional model of cucumber harvesting manipulator by TRIZ. In the functional model, we can understand the relationship between the components of the manipulator system. As shown in Fig. 5, the appearance of chute swing was due to that the slideway cannot limit the roller insufficiently. For reducing the number of drives, the cucumber harvesting manipulator used the under-actuated mechanism. Although this measure reduced the cost and simplified the mechanism, it led to the loss of sufficient limitation of roller from slideway as well. So we need to optimize the mechanism based on this key point.

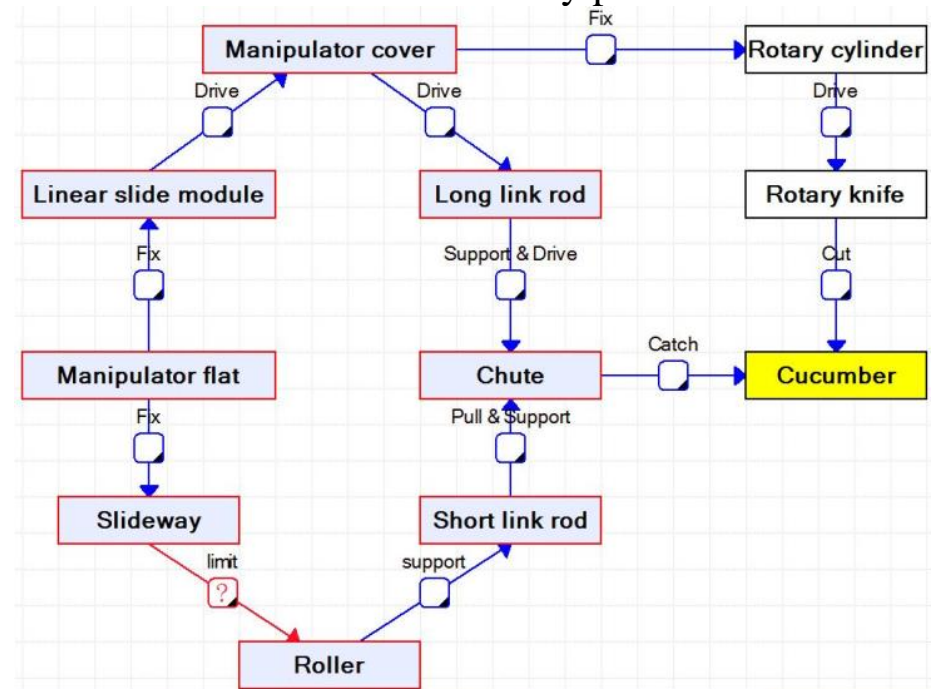

Fig. 5 The functional analysis model cucumber harvesting manipulator by TRIZ

\subsection{Define Technical Contradiction Problem.}

Base on the functional model analysis of the stability problem system, the problem happened between slideway and roller and can be defined as contradiction problem. Based on the design of harvesting manipulator and the rules of TRIZ, the problem can be defined as a technical contradiction: "although the under-actuated mechanism reduce the complication of harvesting manipulator, it also leads to the loss of reliability of mechanism". So according to the description of technical contradiction, we can define it by using 39 engineering parameters. The improved engineering parameter is the "System complexity", and the worsening engineering parameter is "reliability" [6]. After these treatments, the stability problem was transformed into a standard problem.

\subsection{Solve Problem by TRIZ Method.}

In the basis of the analysis above, this paper intends to use the invention principle to solve the technical contradiction. By referring to the conflict matrix, we can find the inventive principles recommended by TRIZ theory as shown in Table 1 . The conflict matrix table recommended that inventive principles are "13 reverse action principle", "35 physical or chemical parameters change principle" and "1 division principle".

Table 1. Conflict matrix (part)

\begin{tabular}{c|c|cc}
\hline Improve parameter & & & 27. System complexity \\
Worsen parameter & & & $\vdots$ \\
\hline$\vdots$ & & & $\vdots$ \\
\hline 36.Reliability & & $\ldots \ldots \ldots$ & $13,35,1$ \\
\hline
\end{tabular}

By careful analysis, the standard solution, "reverse action principle", was used to solve the technical contradiction. In the original design of harvesting manipulator, the roller is free and unlimited. 
So based on the meaning of reverse action principle, we fix the roller to limite its movement. In this way, we get the improvement design scheme, as shown in Fig. 6. After fixing the roller, the under-actuated mechanism turns in to a linkage mechanism. By using this improvment, this paper not noly solves the stability problem of harvesting manipulator, but also leaves out the slideway and roller to reduce the cost.

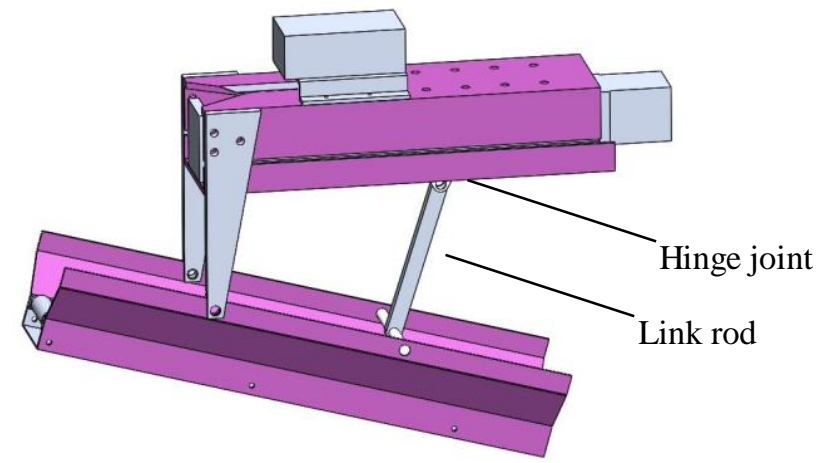

Fig. 6 The specific solution of stability problem by TRIZ

\section{Summary}

For the low stability problem of cucumber harvesting manipulator design, this paper used the theory of TRIZ to solve the problem and purposed the innovative solution to optimize the manipulator. The technical contradiction was defined by the mechanism kinematic simulation and functional model analysis of problem. Finally, not only the harvesting manipulator has optimized by the innovation solution by using TRIZ method, but also the cost and complexity of the harvesting manipulator was reduced greatly.

\section{Acknowledgements}

This work was supported by the National Natural Science Foundation of China (51675011), Innovation Methods Project of Ministry of Science \& Technology (2016IM030400).

\section{References}

[1]. Bac C Wouter, van Henten Eldert J, Hemming Jochen, et al. Harvesting Robots for High-value Crops: State-of-the-art review and Challenges Ahead[J]. Journal of Field Robotics. Vol. 31 (2014) No. 6, p. 888-911.

[2]. Zhang Jianyi, Guo Yanling, Yang Shucai, et al. Innovation design of products based on TRIZ theory[J]. Journal of Machine Design. Vol. 2 (2009) No. 26, p. 35-37.

[3]. HOUSSIN R, COULIBALY A. An approach to solve contradiction problems for the safety integration in innovative design process [J]. Computers in Industry. Vol. 64 (2011) No. 4, p. 398-406.

[4]. Tianxiang Feng, Guohua Gao. Optimization Design of Potted Anthurium Transplanting Manipulator. The previous First International Conference on Materials, Mechanical Engineering and Automation Technology. Guangzhou, May 30-31, 2015, p. 348-353.

[5]. Information on: http://mt.sohu.com/20160628/n456686402.shtml

[6]. Coulibaly S, Hua Zhongsheng, Shi Qin, et al. TRIZ technology forecasting as QFD input within the NPD activities[J]. Chinese Journal of Mechanical Engineering. Vol. 17 (2004) No. 2, p. 284-288. 